\title{
A Fast and Accurate Approach to Analyze Cache Memory Behavior
}

\author{
Xavier Vera, Josep Llosa, Antonio González, and Nerina Bermudo \\ Computer Architecture Department \\ Universitat Politècnica de Catalunya-Barcelona \\ \{xvera, josepll, antonio, nbermudo\}@ac.upc.es
}

\begin{abstract}
In this work, we propose a fast and accurate approach to estimate the solution of Cache Miss Equations (CMEs), which is based on the use of sampling techniques. The results show that only a few seconds are required to analyze most of the SPECfp benchmarks with an error smaller than 0.01 .
\end{abstract}

\section{Introduction}

To take the best advantage of caches, it is necessary to exploit as much as possible the locality that exists in the source code. A locality analysis tool is required in order to identify the sections of the code that are responsible for most penalties and to estimate the benefits of code transformations. Several methods, such as simulators or compilers heuristics, can estimate this information. Unfortunately, simulators are very slow whereas heuristics can be very imprecise.

Our proposal is based on estimating the result of Cache Miss Equations (CMEs) by means of sampling techniques. This technique is very fast and accurate, and the confidence of the error can be chosen.

\section{Overview of CMEs}

CMEs [4] are an analysis framework that describes the behavior of a cache memory. The general idea is to obtain for each memory reference a set of constraints and equations defined over the iteration space that represent the cache misses. These equations make use of the reuse vectors [9]. Each equation describes the iteration points where the reuse is not realized. For more details, the interested reader is referred to the original publications [4, 5].

\subsection{Solving CMEs}

There are two approaches in order to solve CMEs, solve them analitically [4] or checking whether an iteration point is a solution or not. The first approach only works for direct-mapped caches, whereas the second can be used for both direct-mapped and set-associative organizations. 
Traversing the iteration space Given a reference, all the iteration points are tested independently, studying the equations in order: from the equations generated by the shortest reuse vector to the equations generated by the longest one [5]. For this approach, we need to know whether a polyhedron is empty after substituting the iteration point in the equation. This is still a NP-Hard problem, however only $s *$ number_of_points polyhedra must be analyzed.

\section{Sampling}

Our proposal builds upon the second method to solve the CMEs (traversing the iteration space). This approach to solve the CMEs allows us to study each reference in a particular iteration point independently of all other memory references. Based on this property, a small subset of the iteration space can be analyzed, reducing heavily the computation cost. In particular, we use random sampling to select the iteration points to study, and we infer the global miss ratio from them. This sampling technique cannot be applied to a cache simulator. A simulator cannot analyze an isolated reference, since it requires information of all previous references.

\subsection{CMEs Particularization}

We represent a perfectly nested loop of depth $n$ with known bounds as a finite convex polyhedron of the $n$-dimensional iteration space $\mathbb{Z}^{n}$. We are interested in finding the number of misses this loop nest produces (said $m$ ). In order to obtain it, for each reference belonging to the loop nest we define a random variable $(\mathrm{RV})$ that returns the number of misses. Below, we show that this RV follows a Binomial distribution. Thus, we can use statistical techniques (see the full papen [8]) to compute the parameters that describe it.

For each memory instruction, we can define a Bernoulli-RV $X \sim B(p)$ as follows:

$$
\begin{aligned}
& X: \text { Iteration } \quad \text { Space } \longrightarrow \mathbb{R} \\
& \boldsymbol{\imath} \longmapsto\{0,1\}
\end{aligned}
$$

such that $X \equiv 1$ if the memory instruction results in a miss for iteration $\boldsymbol{\imath}, X \equiv 0$ otherwise. Note that $X$ describes the experiment of choosing an iteration point and checking whether the memory instruction produces a miss for it, and $p$ is the probability of success. The value of $p$ is $p=\frac{m}{N}$, where $N$ is the number of iteration points.

Then, we repeat $N$ times the experiment, using a different iteration point in each experiment, obtaining $X_{1}, \ldots, X_{N}$ different RV-variables. We note that:

- All the $X_{i}, i=1 \ldots N$ have the same value of $p$.

- All the $X_{i}, i=1 \ldots N$ are independent.

1 ftp://ftp.ac.upc.es/pub/reports/DAC/2000/UPC-DAC-2000-8.ps.Z 
The variable $Y=\sum X_{i}$ represents the total number of misses in all $N$ experiments. This new variable follows a binomial distribution with parameters $\operatorname{Bin}(\mathrm{N}, \mathrm{p})[3$ and it is defined over all the iteration space. By generating random samples over the iteration space, we can infer the total number of misses.

\subsection{Generating Samples}

The key issues to obtain a good sample are:

- It is important that all the population is represented in the sample.

- The size of the sample.

In our case, we have to keep in mind another constraint: the sample cannot have repeated iteration points (one iteration point cannot result in a miss twice).

To fulfill these requirements, we use Simple Random Sampling 6]. The size of the sample is set according to the required width of the confidence interval and the desired confidence. For instance, for an interval width of 0.05 and a $95 \%$ confidence, 1082 iteration points has to be analyzed.

\section{Evaluation}

CMEs have been implemented for fortran codes through the Polaris Compiler 7 and the Ictineo library [1. We have used our own polyhedra representation [2]. Both direct-mapped and set-associative caches with LRU replacement policy are supported.

\subsection{Performance Evaluation}

Next, we evaluate the accuracy of the proposed method and the speed/accuracy trade-offs for both direct-mapped and set-associative caches.

The loop nests considered are obtained from the SPECfp95, choosing for each program the most time consuming loop nests that in total represent between the $60-70 \%$ of the execution time using the reference input data. The simulation values are obtained using a trace driven cache simulator by instrumenting the program with Ictineo. For the evaluation of the execution time, an Origin2000 has been used.

The CMEs have been generated assuming a 32KB cache of arbitrary associativity.

From our experiments we consider that a $95 \%$ confidence and an interval of 0.05 is a good trade-off between analysis time and accuracy, since the programs are usually analyzed in a few seconds, and never more than 2 minutes. The more accurate configurations require more time because more points are considered.

With a $95 \%$ confidence and an interval width of 0.05 , the absolute difference between the miss ratio and the central point of the confidence interval is usually lower than 0.002 and never higher than 0.01 . 


\section{Set-Associative Caches}

We have also analyzed the SPECfp programs for three different organizations of set-associative caches (2-way, 4-way and 8-way). Although in general the analysis time is higher than for direct mapped caches, it is reasonable (never more than 2 minutes).

As the case of direct mapped caches, the difference between the miss ratio and the empirical estimation for all the programs is usually lower than 0.002 and never higher than 0.01 .

\section{Conclusions}

In this paper we propose the use of sampling techniques to solve CMEs. With these techniques we can perform memory analysis extremely fast independently of the size of the iteration space. For instance, it takes the same time $(3 \mathrm{sec}-$ onds) to analyze a matrix by matrix loop nest of size $100 \times 100$ than one of size 1000x1000. On the contrary it takes 9 seconds to simulate the first case and more than 2 hours to simulate the second.

In our experiments we have found that, using a $95 \%$ confidence and an interval width of 0.05 , the absolute error in the miss ratio was smaller than 0.002 in $65 \%$ of the loops from the SPECfp programs and was never bigger than 0.01 . Furthermore, the analysis time for each program was usually just a few seconds and never more than 2 minutes.

\section{Acknowledgments}

This work has been supported by the ESPRIT project MHAOTEU (EP 24942) and the CICYT project 511/98.

\section{References}

[1] Eduard Ayguadé et al. A uniform internal representation for high-level and instruction-level transformations. UPC, 1995.

[2] Nerina Bermudo, Xavier Vera, Antonio González, and Josep Llosa. An efficient solver for cache miss equations. In IEEE International Symposium on Performance Analysis of Systems and Software, 2000.

[3] M.H. DeGroot. Probability and statistics. Addison-Wesley, 1998.

[4] Somnath Ghosh, Margaret Martonosi, and Sharad Malik. Cache miss equations: an analytical representation of cache misses. In ICS97, 1997.

[5] Somnath Ghosh, Margaret Martonosi, and Sharad Malik. Precise miss analysis for program transformations with caches of arbitrary associativity. In ASPLOS98, 1998.

[6] Moore; McCabe. Introduction to the Practice of Statistics. Freeman \& Co, 1989.

[7] David Padua et al. Polaris developer's document, 1994. 
[8] Xavier Vera, Josep Llosa, Antonio González, and Nerina Bermudo. A fast and accurate approach to analyze cache memory behavior. Technical Report UPCDAC-2000-8, Universitat Politècnica de Catalunya, February 2000.

[9] Michael E. Wolf and Monica S. Lam. A data locality optimizing algorithm. In ACM SIGPLAN91, 1991. 